\title{
Central Limit Theorem for Diffusion Processes in an Anisotropic Random Environment
}

by

\author{
Ernest NIEZNAJ
}

Presented by Stanistaw KWAPIEŃ

Summary. We prove the central limit theorem for symmetric diffusion processes with non-zero drift in a random environment. The case of zero drift has been investigated in e.g. [18], [7]. In addition we show that the covariance matrix of the limiting Gaussian random vector corresponding to the diffusion with drift converges, as the drift vanishes, to the covariance of the homogenized diffusion with zero drift.

1. Introduction. We consider the symmetric diffusion process with a drift in a random environment. Let $\mathbf{x}_{\omega}(t)=\left(x_{\omega}^{1}(t), \ldots, x_{\omega}^{d}(t)\right), t \geq 0$, be the solution of the Itô stochastic differential equation

$$
\left\{\begin{aligned}
d x_{\omega}^{(p)}(t)= & \sum_{q=1}^{d}\left(\frac{1}{2} \partial_{x_{q}} a_{p q}\left(\mathbf{x}_{\omega}(t) ; \omega\right)+v_{q}\right) d t \\
& +\sum_{q=1}^{d} \sigma_{p q}\left(\mathbf{x}_{\omega}(t) ; \omega\right) d w_{q}(t), \\
x_{\omega}^{(p)}(s)= & x,
\end{aligned}\right.
$$

for $p=1, \ldots, d$, where $\mathbf{w}(t)=\left(w_{1}(t), \ldots, w_{d}(t)\right)$ is a $d$-dimensional standard Brownian motion over a probability space $\mathcal{T}:=(\Sigma, \mathcal{A}, Q), \sigma=\left[\sigma_{i j}\right]:=$ $\left[a_{i j}\right]^{1 / 2}$ and $\mathbf{v}=\left(v_{1}, \ldots, v_{d}\right) \neq \mathbf{0}$. Here $\left[a_{i j}(\mathbf{x} ; \omega)\right], \mathbf{x} \in \mathbb{R}^{d}, \omega \in \Omega$, is a symmetric matrix valued stationary random field defined over a probability space $\mathcal{T}_{0}:=(\Omega, \mathcal{B}(\Omega), \mathbb{P})$. We assume that $\Omega$ is a Polish metric space, $\mathcal{B}(\Omega)$ is the Borel $\sigma$-field and $\mathbb{P}$ is a probability measure. Since the field is assumed to be stationary we may and will assume that it is given by $a_{i j}(\mathbf{x} ; \omega):=$

2000 Mathematics Subject Classification: Primary 60K37; Secondary 82D30.

Key words and phrases: diffusions, random fields, effective diffusivity. 
$a_{i j}\left(T_{\mathbf{X}} \omega\right)$, where $\left\{T_{\mathbf{x}}\right\}_{\mathbf{x} \in \mathbb{R}^{d}}$ is a measure preserving group of transformations on $\mathcal{T}_{0}$ that satisfies: $T_{\mathbf{x}}: \Omega \rightarrow \Omega, T_{\mathbf{0}}=I, T_{\mathbf{x}} T_{\mathbf{y}}=T_{\mathbf{x}+\mathbf{y}}, T_{\mathbf{x}}(A) \in \mathcal{B}(\Omega)$, $\mathbb{P}\left[T_{\mathbf{x}}(A)\right]=\mathbb{P}[A]$ for all $\mathbf{x}, \mathbf{y} \in \mathbb{R}^{d}$ and $A \in \mathcal{B}(\Omega)$. We assume that the random matrix $\left[a_{i j}(\omega)\right]_{i, j=1}^{d}$ satisfies the following assumptions:

(A1) $\quad a_{i j}(\omega)=a_{j i}(\omega)$ for $i, j=1, \ldots, d$,

(A2) there exists $\gamma>0$ such that for $\mathbb{P}$-a.s. $\omega \in \Omega$ and $\xi=\left(\xi_{1}, \ldots, \xi_{d}\right)$ $\in \mathbb{R}^{d}$

$$
\gamma\|\xi\|^{2} \leq \sum_{i, j=1}^{d} a_{i j}(\omega) \xi_{i} \xi_{j}
$$

where $\|\xi\|^{2}=\xi_{1}^{2}+\cdots+\xi_{d}^{2}$,

(A3) there exists a deterministic constant $C>0$ such that

$$
\sup _{\omega \in \Omega}\left|a_{i j}(\omega)\right| \leq C
$$

P-a.s. for every $i, j=1, \ldots, d$,

(A4) the mapping $\mathbf{x} \mapsto a_{i j}\left(T_{\mathbf{x}} \omega\right)$ is continuous together with the derivatives up to the second order, and the first derivative is locally Lipschitz for $i, j=1, \ldots, d$, for $\mathbb{P}$-a.s. $\omega$.

For a fixed $\omega$ the process given by (1.1) is a diffusion with the generator

$$
L^{\omega} f(\mathbf{x})=\frac{1}{2} \sum_{i, j=1}^{d} \partial_{x_{i}}\left(a_{i j}\left(T_{\mathbf{x}} \omega\right) \partial_{x_{j}} f(\mathbf{x})\right)+\mathbf{v} \cdot \nabla_{\mathbf{x}} f(\mathbf{x})
$$

where $f \in C_{0}^{2}\left(\mathbb{R}^{d}\right)$. The diffusion in a random environment is a process $\mathbf{x}(t)$, $t \geq 0$, defined over the product probability space $\mathcal{T} \otimes \mathcal{T}_{0}:=(\Omega \times \Sigma, \mathcal{B}(\Omega) \otimes$ $\mathcal{A}, \mathbb{P} \otimes Q)$ given by $\mathbf{x}(t ; \omega, \sigma):=\mathbf{x}_{\omega}(t ; \sigma)$ for any $(\omega, \sigma) \in \Omega \times \Sigma$. It should be stressed that although for a frozen $\omega$, the process $\mathbf{x}_{\omega}(t ; \sigma)$ is Markovian, the process $\mathbf{x}(t)$ need not have the Markov property when considered over the product space.

We are interested in investigating the asymptotic behavior of trajectories of $\mathbf{x}(t)$. It is fairly standard to show, via the ergodic theorem, that $\mathbf{x}(t) / t \rightarrow \mathbf{v}$ a.s., as $t \rightarrow \infty$. The next step is therefore to establish whether the central limit theorem (CLT) holds, i.e. whether the laws of the random vector $(\mathbf{x}(t)-$ $\mathbf{v} t) / \sqrt{t}$ converge to a normal law as $t \rightarrow \infty$.

For $\mathbf{v}=\mathbf{0}$ the problem has already been investigated: see e.g. the paper by Papanicolaou and Varadhan [18], or Kozlov-Zhikov-Oleĭnik [10]. It has been shown that the CLT holds in that case. In this paper we are interested in the effect of the anisotropy of the medium, reflected by the assumption that $\mathbf{v} \neq \mathbf{0}$, on the asymptotic behavior of the particle. Our main result is that the CLT persists in this case (see Theorem 2 below). 
We should stress that although we have diffusive behavior of the particle in both cases of $\mathbf{v}=\mathbf{0}$ and $\mathbf{v} \neq \mathbf{0}$ there are important differences in the proofs caused by the presence of a non-zero drift. The principal object used in the proof of CLT, the so-called corrector field $E(\mathbf{x} ; \omega)$ (see the definition (2.6) below), behaves differently in the two cases. In the isotropic case, i.e. when $\mathbf{v}=\mathbf{0}$, we have sublinear growth of the second moment of the corrector field, i.e. for every $K>0$ and $p=1, \ldots, d$,

$$
\lim _{a \rightarrow \infty} \sup _{|\mathbf{x}| \leq K a} \frac{\left\|E^{(p)}(\mathbf{x})\right\|_{L^{2}}}{a}=0
$$

(see e.g. [18, p. 848]). On the other hand, when the drift in the direction of $\mathbf{v}$ is present then the growth is much slower in the direction parallel to the drift. Namely (see Theorem 1 below), we have

$$
\lim _{a \rightarrow \infty} \sup _{\mathbf{x} \in C(\mathbf{v}, a)} \frac{\left\|E^{(p)}(\mathbf{x})\right\|_{L^{2}}}{a}=0
$$

where $C(\mathbf{v}, a):=\left\{\mathbf{x} \in \mathbb{R}^{d}:|\mathbf{x} \cdot \mathbf{v}| \leq a^{2} K,\left|P_{\mathbf{v}}(\mathbf{x})\right| \leq a K\right\}$ and $P_{\mathbf{v}}(\mathbf{x})$ denotes the complement of the orthogonal projection along the direction of $\mathbf{v}$.

To prove (1.2) we use the Harnack inequality and methods of partial differential equations. In Section 4 we show that (1.2) implies CLT (Theorem 2). Additionally in Theorem 3 we prove that the covariance matrix of the limiting normal random vector corresponding to the diffusion with $\mathbf{v} \neq \mathbf{0}$ converges, as $\mathbf{v} \rightarrow \mathbf{0}$, to the covariance of the homogenized diffusion with $\mathbf{v}=\mathbf{0}$.

2. Preliminaries and the statement of the main theorems. Let $L^{2}(\Omega)$ denote the Hilbert space of all square integrable random variables over the probability space $\mathcal{T}_{0}$. For $F, G \in L^{2}(\Omega)$ we denote by $(F, G)_{L^{2}}$ the standard scalar product, i.e.

$$
(F, G)_{L^{2}}:=\int F G d \mathbb{P} .
$$

The norm is then given by $\|F\|_{L^{2}}:=\sqrt{(F, F)}, F \in L^{2}(\Omega)$. We define the unitary group by $U^{\mathbf{x}} F=F\left(T_{\mathbf{x}} \omega\right), \mathbf{x} \in \mathbb{R}^{d}$. Its generators are given by

$$
D_{k} F(\omega)=\left.\frac{\partial}{\partial x_{k}} F\left(T_{\mathbf{x}} \omega\right)\right|_{\mathbf{x}=0}, \quad k=1, \ldots, d,
$$

for $F \in \mathcal{D}\left(D_{k}\right)$, where the differentiation is understood in the $L^{2}(\Omega)$ sense. By Corollary 1.1.6 of [2] the infinitesimal generators are closed and densely defined in $L^{2}(\Omega)$. Let $C_{b}^{m}(\Omega)$ denote the space of all $F \in L^{2}(\Omega)$ which have $m$ deterministically bounded derivatives. Since $\left\{T_{\mathbf{x}}\right\}_{\mathbf{x} \in \mathbb{R}^{d}}$ is measure 
preserving, for $F, G \in \mathcal{D}\left(D_{k}\right)$ and for any $k=1, \ldots, d$ we have

$$
\int_{\Omega} D_{k} F G d \mathbb{P}=-\int_{\Omega} F D_{k} G d \mathbb{P} .
$$

Substituting $G=1$ we obtain

$$
\int_{\Omega} D_{k} F d \mathbb{P}=0
$$

(see also [14, p. 16] for more details).

Using $\mathbf{x}_{\omega}(t)$ we introduce (for fixed $\omega \in \Omega$ ) the environment process $\xi_{t}$ defined by

$$
\xi_{t}=T_{\mathbf{x}_{\omega}(t)}(\omega)
$$

for $t \geq 0$ with state space $\Omega$. For $t \geq 0$ let $P^{t} F(\omega)=\int F\left(T_{\mathbf{x}} \omega\right) p^{\omega}(t, 0, \mathbf{x}) d \mathbf{x}$ where $p^{\omega}(t, x, y)$ is a transition of probability density corresponding to diffusion (1.1), $F \in B(\Omega)$. Since $\int p^{\omega}(t, \mathbf{x}, \mathbf{y}) d \mathbf{x}=\int p^{\omega}(t, \mathbf{x}, \mathbf{y}) d \mathbf{y}$ it follows that $\int P^{t} F d \mathbb{P}=\int F d \mathbb{P}$ for $F \in B(\Omega)$ and $\left(P^{t}\right)$ can be extended to a $C_{0}$ semigroup on $L^{2}(\Omega)$. It turns out that $\xi_{t}$ is a Markov process with $\left(P^{t}\right)$ as its transition of probability semigroup (see [6, p. 104]), and its generator equals

$$
\mathcal{L} F(\omega)=\frac{1}{2} \sum_{i, j=1}^{d} D_{i}\left(a_{i j}(\omega) D_{j} F(\omega)\right)+\mathbf{v} \cdot \nabla F(\omega)
$$

where $\nabla=\left(D_{1}, \ldots, D_{d}\right)$ and $F \in C^{2}(\Omega)$, which is a core of $\mathcal{L}$.

Next we solve the resolvent equation in $L^{2}(\Omega)$ :

$$
\lambda E_{\lambda}^{(p)}-\mathcal{L} E_{\lambda}^{(p)}=\widetilde{v}^{(p)}
$$

with $\lambda>0$, where

$$
\widetilde{v}^{(p)}(\omega)=\frac{1}{2} \sum_{i=1}^{d} D_{i} a_{i p}(\omega)
$$

for $p=1, \ldots, d$. Multiplying $(2.3)$ by $E_{\lambda}^{(p)}$ and integrating over $\Omega$ we get

$$
\begin{array}{rl}
\lambda \int_{\Omega}\left[E_{\lambda}^{(p)}\right]^{2} & \mathbb{P}(d \omega)-\frac{1}{2} \sum_{i, j=1}^{d} \int_{\Omega} D_{i}\left(a_{i j}(\omega) D_{j} E_{\lambda}^{(p)}\right) E_{\lambda}^{(p)} \mathbb{P}(d \omega) \\
& -\int_{\Omega} \mathbf{v} \cdot \nabla E_{\lambda}^{(p)} E_{\lambda}^{(p)} \mathbb{P}(d \omega)=\frac{1}{2} \sum_{i=1}^{d} \int_{\Omega} D_{i} a_{i p}(\omega) E_{\lambda}^{(p)} \mathbb{P}(d \omega) .
\end{array}
$$

Since

$$
\int_{\Omega} D_{i} E_{\lambda}^{(p)} E_{\lambda}^{(p)} \mathbb{P}(d \omega)=-\int_{\Omega} E_{\lambda}^{(p)} D_{i} E_{\lambda}^{(p)} \mathbb{P}(d \omega), \quad i=1, \ldots, d,
$$


this term equals zero. Thus we can rewrite (2.4) in the form of the so-called energy inequality

$$
\begin{aligned}
\varlimsup_{\lambda \rightarrow 0+} \lambda\left\|E_{\lambda}^{(p)}\right\|_{L^{2}}^{2}+\frac{1}{2} \sum_{i, j=1}^{d} \int_{\Omega} a_{i j}(\omega) D_{j} E_{\lambda}^{(p)} D_{i} E_{\lambda}^{(p)} \mathbb{P}(d \omega) \\
\leq-\frac{1}{2} \sum_{i=1}^{d} \int_{\Omega} a_{i p}(\omega) D_{i} E_{\lambda}^{(p)} \mathbb{P}(d \omega) .
\end{aligned}
$$

From (2.5) we conclude that

$$
\sup _{0<\lambda<1}\left\|D_{i} E_{\lambda}^{(p)}\right\|_{L^{2}} \leq C \quad \text { for } i=1, \ldots, d,
$$

where $C$ is a constant independent of $\lambda$ and $\mathbf{v}$. Hence there exists a sequence $\lambda_{n} \rightarrow 0$ as $n \rightarrow \infty$ such that

$$
e_{k}^{(p)}=\lim _{n \rightarrow \infty} D_{k} E_{\lambda_{n}}^{(p)} \quad \text { for } k=1, \ldots, d
$$

in the weak $L^{2}$ sense. Next we define the following random field, called the corrector field:

$$
E(\mathbf{x} ; \omega)=\left(E^{(1)}(\mathbf{x} ; \omega), \ldots, E^{(d)}(\mathbf{x} ; \omega)\right), \quad(\mathbf{x} ; \omega) \in \mathbb{R}^{d} \times \Omega,
$$

where

$$
E^{(p)}(\mathbf{x} ; \omega):=\sum_{k=1}^{d} \int_{0}^{1} e_{k}^{(p)}\left(T_{t \mathbf{x}} \omega\right) x_{k} d t, \quad p=1, \ldots, d,
$$

and $E^{(p)}(\mathbf{0} ; \omega)=0$.

Proposition 1. The following conditions are satisfied:

(i) $E^{(p)}(\mathbf{x} ; \cdot) \in L^{2}$

(ii) $\partial_{x_{j}} E^{(p)}(\mathbf{x} ; \omega)=e_{j}^{(p)}\left(T_{\mathbf{x}} \omega\right)$ for $j=1, \ldots, d$,

(iii) for any continuously differentiable function $\phi: \mathbb{R}^{d} \times \Omega \rightarrow \mathbb{R}$ such that $\phi(\cdot, \omega)$ is compactly supported for every $\omega \in \Omega$ and $\phi(\mathbf{x}, \cdot) \in L^{2}$ for any $\mathrm{x} \in \mathbb{R}^{d}$ we have

$$
\begin{aligned}
\frac{1}{2} \sum_{i, j=1}^{d} \iint_{\mathbb{R}^{d}} a_{\Omega}\left(T_{\mathbf{x}} \omega\right) \partial_{x_{j}} E^{(p)}(\mathbf{x} ; \omega) \partial_{x_{i}} \phi(\mathbf{x}, \omega) d \mathbf{x} \mathbb{P}(d \omega) & \\
& +\sum_{i=1}^{d} \iint_{\mathbb{R}^{d}} v_{i} E^{(p)}(\mathbf{x} ; \omega) \partial_{x_{i}} \phi(\mathbf{x}, \omega) d \mathbf{x} \mathbb{P}(d \omega) \\
= & -\frac{1}{2} \sum_{i=1}^{d} \iint_{\mathbb{R}^{d}} a_{i p}\left(T_{\mathbf{x}} \omega\right) \partial_{x_{i}} \phi(\mathbf{x}, \omega) d \mathbf{x} \mathbb{P}(d \omega)
\end{aligned}
$$


Proof. From (2.6) it follows that $\partial_{x_{i}} E^{(p)}(\mathbf{x} ; \omega)=e_{i}^{(p)}\left(T_{\mathbf{x}} \omega\right)$ so (i) and (ii) hold. For (iii) see e.g. [18, proof of Theorem 2].

In the present paper we use the following version of the ergodic theorem (see [11, Theorem 2.13, p. 210] and [9, Proposition 6, p. 103]).

Proposition 2. Suppose $f \in L^{1}(\Omega)$ and $\phi \in C_{0}\left(\mathbb{R}^{d}\right)$. Then

$$
\lim _{a \rightarrow \infty} \frac{1}{a^{d+1}} \int_{\mathbb{R}^{d}} f\left(T_{\mathbf{x}} \omega\right) \phi\left(\frac{x_{1}}{a^{2}}, \frac{x_{2}}{a}, \ldots, \frac{x_{d}}{a}\right) d \mathbf{x}=\mathbb{E}[f] \int_{\mathbb{R}^{d}} \phi(\mathbf{x}) d \mathbf{x}
$$

$\mathbb{P}$-a.s. and in the $L^{1}$ norm.

Now we can formulate the main results.

THEOREM 1. The random field $E(\mathbf{x} ; \omega)$ satisfies

$$
\lim _{a \rightarrow \infty} \sup _{\mathbf{x} \in C(\mathbf{v}, a)} \frac{\left\|E^{(p)}(\mathbf{x})\right\|_{L^{2}}}{a}=0
$$

We show in Section 4 the following corollary of Theorem 1.

THEOREM 2. Suppose that the random matrix-valued field $\left[a_{i j}\right]$ satisfies the assumptions (A1)-(A4). Then the sequence of the laws corresponding to the random vectors $(\mathbf{x}(t)-\mathbf{v} t) / \sqrt{t}$ converges weakly, as $t \rightarrow \infty$, to the law of a normal random vector of mean $\mathbf{0}$ with covariance matrix $D^{*}$.

REMARK 1. A more detailed description of $D^{*}$ will be given in Section 4 (see Remark 2).

Multiplying both sides of (2.3) by $\phi \in C_{b}^{1}(\Omega)$, integrating over $\Omega$ and letting $\lambda \rightarrow 0$ we obtain

$$
\frac{1}{2} \sum_{i, j=1}^{d} \int_{\Omega} a_{i j} e_{i}^{(p)} D_{j} \phi d \mathbb{P}-\sum_{i=1}^{d} \int_{\Omega} v_{i} e_{i}^{(p)} \phi d \mathbb{P}=-\frac{1}{2} \sum_{i=1}^{d} \int_{\Omega} a_{i p} D_{i} \phi d \mathbb{P} .
$$

Theorem 3. (i) There is a unique solution $e_{i}^{(p)}, i=1, \ldots, d$, of (2.10) such that $\int e_{i}^{(p)} D_{j} \phi d \mathbb{P}=\int e_{j}^{(p)} D_{i} \phi d \mathbb{P}$. We denote this solution by $e_{i}^{(p)}(\mathbf{v})$.

(ii) We have

$$
\lim _{\mathbf{v} \rightarrow \mathbf{0}}\left(e_{1}^{(p)}(\mathbf{v}), \ldots, e_{d}^{(p)}(\mathbf{v})\right)=\left(e_{1}^{(p)}(\mathbf{0}), \ldots, e_{d}^{(p)}(\mathbf{0})\right)
$$

in the $L^{2}$ sense. 
3. Proof of Theorem 1. From the definition of $E(\mathbf{x} ; \omega)$ it follows that it satisfies, in the weak p.d.e. sense, the following equation:

$$
\begin{aligned}
\frac{1}{2} \sum_{i, j=1}^{d} \partial_{x_{j}}\left(a_{i j}\left(T_{\mathbf{x}} \omega\right) \partial_{x_{j}} E^{(p)}(\mathbf{x})\right)+\sum_{i=1}^{d} v_{i} \partial_{x_{i}} E^{(p)}(\mathbf{x}) & \\
& =-\frac{1}{2} \sum_{i=1}^{d} \partial_{x_{i}} a_{i p}\left(T_{\mathbf{x}} \omega\right)
\end{aligned}
$$

P-a.s. Let $\chi^{(p)}(t, \mathbf{x}):=E^{(p)}(\mathbf{x}+\mathbf{v} t)$. It has the following properties:

$$
\partial_{t} \chi^{(p)}(t, \mathbf{x})=\mathbf{v} \cdot \nabla_{\mathbf{x}} E^{(p)}(\mathbf{x}+\mathbf{v} t), \quad \partial_{x_{j}} \chi^{(p)}(t, \mathbf{x})=\partial_{x_{j}} E^{(p)}(\mathbf{x}+\mathbf{v} t),
$$

hence

$$
\begin{aligned}
& \partial_{t} \chi^{(p)}(t, \mathbf{x}) \\
& \quad=-\frac{1}{2} \sum_{i, j=1}^{d} \partial_{x_{i}}\left(a_{i j}\left(T_{\mathbf{x}} \omega\right) \partial_{x_{j}} E^{(p)}(\mathbf{x}+\mathbf{v} t)\right)-\frac{1}{2} \sum_{i=1}^{d} \partial_{x_{i}} a_{i p}\left(T_{\mathbf{x}} \omega\right) .
\end{aligned}
$$

Let $y^{(p)}(t, \mathbf{x}):=x_{p}+\chi^{(p)}(t, \mathbf{x})$. Notice that $\partial_{x_{q}} y^{(p)}(t, \mathbf{x})=\delta_{p q}+\partial_{x_{q}} \chi^{(p)}(t, \mathbf{x})$, hence $y^{(p)}(t, \mathbf{x})$ is a weak solution of the following inverse time parabolic p.d.e.:

$$
\partial_{t} y^{(p)}(t, \mathbf{x})+\frac{1}{2} \sum_{i, j=1}^{d} \partial_{x_{i}}\left(a_{i j}\left(T_{\mathbf{x}} \omega\right) \partial_{x_{j}} y^{(p)}(t, \mathbf{x})\right)=0 .
$$

It is now easy to see that (2.9) is equivalent to the condition

$$
\lim _{a \rightarrow \infty} \sup _{|t| \leq K a^{2},|\mathbf{x}| \leq K a} \frac{\left\|\chi^{(p)}(t, \mathbf{x})\right\|_{L^{2}}}{a}=0 .
$$

We define the scaled functions

$$
\chi_{a}^{(p)}(t, \mathbf{x})=a^{-1} \chi^{(p)}\left(a^{2} t, a \mathbf{x}\right), \quad y_{a}^{(p)}(t, \mathbf{x})=a^{-1} y^{(p)}\left(a^{2} t, a \mathbf{x}\right),
$$

for $a>0$. Hence the function $y_{a}^{(p)}(t, \mathbf{x})$ satisfies the inverse time parabolic p.d.e.

$$
\partial_{t} y_{a}^{(p)}(t, \mathbf{x})=-\frac{1}{2} \sum_{i, j=1}^{d} \partial_{x_{i}}\left(a_{i j}\left(a^{2} \mathbf{v} t+a \mathbf{x}\right) \partial_{x_{j}} y_{a}^{(p)}(t, \mathbf{x})\right) .
$$

The condition (3.3) (and in consequence $(2.9)$ ) will be proven if we can show that

$$
\lim _{a \rightarrow \infty} \sup _{|t| \leq K,|\mathbf{x}| \leq K}\left\|\chi_{a}^{(p)}(t, \mathbf{x})\right\|_{L^{2}}=0
$$

for any $K>0$.

We prove (3.5) in several steps. We use the following elementary lemma (see e.g. [19, pp. 114-116]. 
Lemma 1. Assume that on a probability space $(\Omega, \mathcal{F}, \mathbb{P})$ we have two sequences of random variables, $\left\{X_{n}\right\}$ and $\left\{Z_{n}\right\}, n \geq 1$. If the following conditions hold:

(i) $\lim _{n \rightarrow \infty} X_{n}=0, \mathbb{P}$-a.s.,

(ii) $0 \leq X_{n} \leq Z_{n}, n \geq 1$,

(iii) $Z_{n} \rightarrow Z$ in the $L^{1}$-norm, then

$$
\lim _{n \rightarrow \infty}\left\|X_{n}\right\|_{L^{1}}=0
$$

The first step is to show that

$$
\sup _{(t, \mathbf{x}) \in \Omega_{T, R}}\left|y_{a}^{(p)}(t, \mathbf{x})-x_{p}\right| \rightarrow 0, \quad \mathbb{P} \text {-a.s. }
$$

where $\Omega_{T, R}:=[0 \leq t \leq T] \times B_{R}(0)$. This fact is a consequence of the Harnack inequality (see (3.9)) and

$$
\lim _{a \rightarrow \infty} \iint_{\Omega_{T, R}}\left[y_{a}^{(p)}(t, \mathbf{x})-x_{p}\right] \phi(t, \mathbf{x}) d t d \mathbf{x}=0, \quad \mathbb{P} \text {-a.s. }
$$

where $\phi \in C_{0}\left(\Omega_{T, R}\right)$. The equality (3.7) will be proven at the end of the section.

The second step is to show that

$$
\mathbb{E}\left[\sup _{(t, \mathbf{x}) \in \Omega_{T, R}}\left|y_{a}^{(p)}(t, \mathbf{x})-x_{p}\right|^{2}\right]=0
$$

as $a \rightarrow \infty$. This will be a consequence of the first step and Lemma 1. With the help of this fact we can easily estimate the expression

$$
\mathbb{E}\left[\left|y_{a}^{(p)}(t, \mathbf{x})-x_{p}\right|^{2}\right] \leq \mathbb{E}\left[\sup _{(s, \mathbf{z}) \in \Omega_{T, R}}\left|y_{a}^{(p)}(s, \mathbf{z})-x_{p}\right|^{2}\right], \quad \forall(t, \mathbf{x}) \in \Omega_{T, R},
$$

and take the limit as $a \rightarrow \infty$.

We will show that

$$
\lim _{a \rightarrow \infty} \iint_{\Omega_{T, R}}\left|\nabla_{\mathbf{x}} y_{a}^{(p)}(t, \mathbf{x})\right|^{2} d t d \mathbf{x}<\infty
$$

(cf. (3.12) below). Recall that $\nabla_{\mathbf{x}} y_{a}^{(p)}(t, \mathbf{x})=\mathbf{e}_{p}+\nabla_{\mathbf{x}} \chi^{(p)}\left(a^{2} t, a \mathbf{x}\right)$.

We will also prove that

$$
\lim _{a \rightarrow \infty} \iint_{\Omega_{T, R}}\left|y_{a}^{(p)}(t, \mathbf{x})\right| d t d \mathbf{x} \leq C_{1}
$$

and

$$
\sup _{(t, \mathbf{x}) \in \Omega_{T^{\prime}, R^{\prime}}}\left|y_{a}^{(p)}(t, \mathbf{x})\right| \leq C_{2} \iint_{\Omega_{T, R}}\left|y_{a}^{(p)}(t, \mathbf{x})\right| d t d \mathbf{x}, \quad \mathbb{P} \text {-a.s. }
$$

Now we begin the proof of (3.6). We use the Harnack inequality for subsolutions of parabolic p.d.e. proved by Moser in [13]. Since $\varphi(\mathbf{x})=$ 
$\sqrt{\mathbf{x}^{2}+1}$ is a twice differentiable convex function, the function $u_{a}^{(p)}(t, \mathbf{x})=$ $\sqrt{y_{a}^{(p)}(t, \mathbf{x})^{2}+1}$ is a subsolution of (3.4) (see [13, p. 117 for definition]). Using the triangle inequality and Theorem 3, p. 113 of [13] applied for (3.4) and the subsolution $u_{a}^{(p)}$ we have

$$
\sup _{(t, \mathbf{x}) \in \Omega_{T^{\prime}, R^{\prime}}}\left|y_{a}^{(p)}(t, \mathbf{x})-x_{p}\right| \leq C\left(\iint_{\Omega_{T, R}}\left[u_{a}^{(p)}(t, \mathbf{x})\right]^{2} d t d \mathbf{x}\right)^{1 / 2}
$$

where $\bar{\Omega}_{T^{\prime}, R^{\prime}} \subset \Omega_{T, R}$ and $C$ is an absolute constant that only depends on $T^{\prime}, T, R^{\prime}, R$ but not on $\omega$. We use (3.9) in order to show that the term on the left hand side of this inequality is bounded (see (3.10) below). A consequence of the Harnack inequality is the following result (see also [13, p. 109]): the family $\left\{y_{a}^{(p)}\right\}_{a>0}$ is equicontinuous on $\Omega_{T, R}$. The fact that the family $\left\{y_{a}^{(p)}\right\}_{a>0}$ is also bounded allows us to use the Arzelà-Ascoli theorem on $\Omega_{T, R}$. The last two statements and the compactness of $\left\{y_{a}^{(p)}\right\}_{a>0}$ imply (3.6).

We show the following facts:

$$
\lim _{a \rightarrow \infty} \iint_{\Omega_{T, R}}\left[u_{a}^{(p)}(t, \mathbf{x})\right]^{2} d t d \mathbf{x}<\infty, \quad \mathbb{P} \text {-a.s. }
$$

and

$$
\lim _{a \rightarrow \infty} \mathbb{E} \iint_{\Omega_{T, R}}\left[u_{a}^{(p)}(t, \mathbf{x})\right]^{2} d t d \mathbf{x}<\infty .
$$

Now we start the proof of (3.10). Using the Fubini's theorem, the Poincaré inequality (for fixed $t$ ) and the definition of $u_{a}^{(p)}$ we get the estimate (cf. [3, p. 768])

$$
\begin{aligned}
& \iint_{\Omega_{2 T, 2 R}}\left[u_{a}^{(p)}(t, \mathbf{x})\right]^{2} d t d \mathbf{x}=\iint_{\Omega_{2 T, 2 R}}\left(\left[y_{a}^{(p)}(t, \mathbf{x})\right]^{2}+1\right) d t d \mathbf{x} \\
& \leq C \iiint_{\Omega_{2 T, 2 R}}\left|\nabla_{\mathbf{x}} y_{a}^{(p)}(t, \mathbf{x})\right|^{2} d t d \mathbf{x}+C \int_{0}^{2 T} d t\left(\int_{B_{2 R}} y_{a}^{(p)}(t, \mathbf{x}) d \mathbf{x}\right)^{2}+C
\end{aligned}
$$

where $C$ is an absolute constant. The right hand side of the above inequality will be used as the upper estimate in part (ii) of Lemma 1. Denote by $M(R)$ the first and by $N(R)$ the second term on the right hand side of (3.12). Since $\partial_{x_{k}} y_{a}^{(p)}(t, \mathbf{x})=\left(\partial_{x_{k}} y^{(p)}\right)\left(a^{2} t, a \mathbf{x}\right)$ we have

$$
\iint_{\Omega_{2 T, 2 R}}\left[\partial_{x_{k}} y_{a}^{(p)}(t, \mathbf{x})\right]^{2} d t d \mathbf{x}=\iint_{\Omega_{2 T, 2 R}}\left[\left(\partial_{x_{k}} y^{(p)}\right)\left(a^{2} t, a \mathbf{x}\right)\right]^{2} d t d \mathbf{x} .
$$

Recall that $e_{k}^{(p)}=\partial_{x_{k}} y^{(p)}$ and use the ergodic theorem in the form of Propo- 
sition 2 for $M(R)$ to obtain

$$
\lim _{a \rightarrow \infty} \iint_{\Omega_{2 T, 2 R}}\left[\partial_{x_{k}} y^{(p)}\left(a^{2} t, a \mathbf{x}\right)\right]^{2} d t d \mathbf{x}=\left|\Omega_{2 T, 2 R}\right| \mathbb{E}\left(e_{k}^{(p)}\right)^{2}
$$

$\mathbb{P}$-a.s. and in the $L^{1}$-norm. The second term $N(R)$ can be written as

$$
\int_{B_{2 R}} y_{a}^{(p)}(t, \mathbf{x}) d \mathbf{x}=\int_{B_{2 R}} y_{a}^{(p)}(0, \mathbf{x}) d \mathbf{x}+\int_{0}^{t}\left(\frac{d}{d s} \int_{B_{2 R}} y_{a}^{(p)}(s, \mathbf{x}) d \mathbf{x}\right) d s .
$$

As in the previous expression, denote by $I$ the first and by $I I$ the second term on the right hand side of (3.13). Then

$$
I=a^{-1} \int_{B_{2 R}} E^{(p)}(a \mathbf{x}) d \mathbf{x} .
$$

Thanks to (3.4) we have

$$
\begin{aligned}
I I & =\int_{0}^{t} d s \int_{B_{2 R}} \partial_{s} y_{a}^{(p)}(s, \mathbf{x}) d \mathbf{x} \\
& =-\sum_{i, j=1}^{d} \int_{0}^{t} d s \int_{B_{2 R}} \partial_{x_{i}}\left[a_{i j}\left(a^{2} s+a \mathbf{x}\right) \partial_{x_{j}} y_{a}^{(p)}(s, \mathbf{x})\right] d \mathbf{x} \\
& =-\sum_{i, j=1}^{d} \int_{0}^{t} d s \int_{S_{2 R}} a_{i j}\left(a^{2} s+a \mathbf{x}\right) \partial_{x_{j}} y_{a}^{(p)}(s, \mathbf{x}) \frac{x_{i}}{|\mathbf{x}|} d S .
\end{aligned}
$$

We can estimate $N(R)$ by

$$
\int_{0}^{2 T} d t\left(\int_{B_{2 R}} y_{a}^{(p)}(t, \mathbf{x}) d \mathbf{x}\right)^{2} \leq 2\left(\int_{0}^{2 T} I^{2} d t+\int_{0}^{2 T} I I^{2} d t\right) .
$$

Let us rewrite $I$ in the following form:

$$
I=a^{-1} \int_{B_{2 R}}\left(\int \frac{d}{d u} E^{(p)}(a \mathbf{x} u) d u\right) d \mathbf{x}=\sum_{j=1}^{d} \int_{B_{2 R}} x_{j} d \mathbf{x} \int_{0}^{1} e_{j}^{(p)}(a \mathbf{x} u) d u .
$$

Now we can use the estimate

$$
I^{2} \leq C R^{2} \int_{0}^{1} d u \int_{B_{2 R}}\left[e_{j}^{(p)}(a \mathbf{x} u)\right]^{2} d \mathbf{x}
$$

Set

$$
\mathcal{E}(a u):=\int_{B_{2 R}}\left[e_{j}^{(p)}\right]^{2}(a \mathbf{x} u) d \mathbf{x}
$$


From the ergodic theorem it follows that

$$
\int_{B_{2 R}}\left[e_{j}^{(p)}\right]^{2}(b \mathbf{x}) d \mathbf{x} \rightarrow \mathbb{E}\left[e_{j}^{(p)}\right]^{2}\left|B_{2 R}\right|
$$

$\mathbb{P}$-a.s., as $b \rightarrow \infty$. Denote the right hand side of $(3.14)$ by $\mathcal{E}(b ; \omega)$ and the left hand side by $\mathcal{E}(\infty)$. From this equation it follows that there exists $b_{0}$ such that for $b \geq b_{0}(\omega)$ we have

$$
\mathcal{E}(b) \leq \mathcal{E}(\infty)+1
$$

Next note that

$$
\begin{aligned}
I^{2} & \leq C R^{2} \int_{0}^{1} \mathcal{E}(a u) d u=C R^{2} \int_{1 \geq a u \geq b_{0}(\omega)} \mathcal{E}(a u) d u+C R^{2} \int_{0 \leq a u \leq b_{0}(\omega)} \mathcal{E}(a u) d u \\
& \leq C R^{2} \int(\mathcal{E}(\infty)+1)+C R^{2} \int_{0 \leq a u \leq b_{0}(\omega)} \int_{B_{2 R}}\left[e_{j}^{(p)}\right]^{2}(a u \mathbf{x}) d \mathbf{x} \\
& \leq C R^{2}(\mathcal{E}(\infty)+1)+C R^{2} \int_{0 \leq a y \leq b_{0}(\omega)} \frac{1}{(a y)^{d}} \int_{B_{2 a u R}}\left[e_{j}^{(p)}\right]^{2}(\mathbf{y}) d \mathbf{y} \\
& \leq C R^{2}(\mathcal{E}(\infty)+1)+C R^{2} \sup _{B_{2 b_{0} R}\left|e_{j}^{(p)}\right|^{2}\left|2 B_{R}\right| .}
\end{aligned}
$$

From the ergodic theorem we conclude that the above estimate holds $\mathbb{P}$-a.s. Now we prove that we also have $L^{1}$-convergence (cf. (3.11)), i.e.

$$
\lim _{a \rightarrow \infty} \mathbb{E}\left|\int_{0}^{1} d u \int_{B_{2 R}}\left[e_{j}^{(p)}(a u \mathbf{x})\right]^{2} d \mathbf{x}-\mathbb{E}\left[e_{j}^{(p)}\right]^{2}\right| B_{2 R}||=0
$$

From the mean ergodic theorem it follows that

$$
\lim _{b \rightarrow \infty} \mathbb{E}\left|\int_{B_{2 R}}\left[e_{j}^{(p)}(b \mathbf{x})\right]^{2} d \mathbf{x}-\mathcal{E}(\infty)\right|=0 .
$$

Denote the left hand side of (3.15) by $S(a)$ and the left hand side of (3.16) by $R(b)$. For any $\varepsilon>0$ there exists $b_{0}$ such that for every $b \geq b_{0}$ we have $R(b)<\varepsilon$. Hence

$$
\begin{aligned}
S(b) & \leq \int_{b_{0} \leq u a \leq 1} R(u a) d u+\mathbb{E}\left|\int_{0 \leq a u \leq b_{0}} d u\left(\int_{B_{2 R}}\left[e_{j}^{(p)}(a u \mathbf{x})\right]^{2} d \mathbf{x}-\mathcal{E}(\infty)\right)\right| \\
& \leq \varepsilon+2\left|B_{2 R}\right| \mathbb{E}\left(e_{j}^{(p)}\right)^{2} \frac{b_{0}}{a}
\end{aligned}
$$

which vanishes as $a \rightarrow \infty$. 
Now we estimate the term $I I$ :

$$
\begin{aligned}
& \int_{0}^{2 T} I I^{2} d t \\
& \leq C \sum_{i, j=1}^{d} \int_{0}^{2 T} d t\left(\int_{0}^{2 T} d s \int_{S_{2 R}}\left|a_{i j}\left(a^{2} s+a \mathbf{x}\right)\right|\left|e_{j}^{(p)}\left(a^{2} s+a \mathbf{x}\right)\right| S(d \mathbf{x})\right)^{2} \\
& \leq C \sum_{i, j=1}^{d} \int_{0}^{2 T} d s \int_{S_{2 R}}\left|a_{i j}\left(a^{2} s+a \mathbf{x}\right)\right|^{2}\left|e_{j}^{(p)}\left(a^{2} s+a \mathbf{x}\right)\right|^{2} S(d \mathbf{x})=: \widetilde{I I}(R) .
\end{aligned}
$$

All the above calculations have led us to the following estimate:

$$
\iint_{\Omega_{2 T, 2 R}}\left[u_{a}^{(p)}(t, \mathbf{x})\right]^{2} d t d \mathbf{x} \leq C^{\prime}(A(R)+B(R))
$$

where $C^{\prime}$ is a constant, and $A(R)$ and $B(R)$ denote the two terms on the right hand side of (3.12). Let $R_{0}>0$ be fixed; then

$$
\begin{aligned}
R_{0} \iint_{\Omega_{2 T, 2 R_{0}}}\left[u_{a}^{(p)}(t, \mathbf{x})\right]^{2} d t d \mathbf{x} \leq \int_{R_{0}}^{2 R_{0}} d R \iiint_{\Omega_{2 T, 2 R}}\left[u_{a}^{(p)}(t, \mathbf{x})\right]^{2} d t d \mathbf{x} \\
\leq C^{\prime} \int_{R_{0}}^{2 R_{0}}\left(A(R)+I^{2}(R)+\widetilde{I I}(R)\right) d R \\
\leq C^{\prime} R_{0} A\left(2 R_{0}\right)+C^{\prime} C_{2 R_{0}} R_{0}+C^{\prime} \int_{R_{0}}^{2 R_{0}} \widetilde{I I}(R) d R .
\end{aligned}
$$

Set $f_{i j}\left(a^{2} s+a \mathbf{x}\right):=a_{i j}\left(a^{2} s+a \mathbf{x}\right) e_{j}\left(a^{2}+a \mathbf{x}\right)$ and note that $f_{i j}$ is also a time-space stationary random field. The right hand side of (3.19) can be written as

$$
C^{\prime} R_{0} A\left(2 R_{0}\right)+C^{\prime} C_{2 R_{0}} R_{0}+C^{\prime} \sum_{i, j=1}^{d} \int_{0}^{2 T} d s \int_{B_{4 R_{0}} \backslash B_{2 R_{0}}} f_{i j}\left(a^{2} s+a \mathbf{x}\right) d \mathbf{x} .
$$

A consequence of these estimates is the upper bound

$$
\begin{aligned}
\iint_{\Omega_{2 T, 2 R}}\left[u_{a}^{(p)}(t, \mathbf{x})\right]^{2} d t d \mathbf{x} \leq & C^{\prime} A\left(2 R_{0}\right)+C^{\prime} C_{2 R_{0}} \\
& +\frac{C^{\prime}}{R_{0}} \sum_{i, j=1}^{d} \int_{0}^{2 T} d s \int_{B_{4 R_{0} \backslash B_{2 R_{0}}} f_{i j}\left(a^{2} s+a \mathbf{x}\right) d \mathbf{x} .}
\end{aligned}
$$

By the ergodic theorem the third term on the right hand side of this inequality converges both in the $L^{1}$-norm and $\mathbb{P}$-a.s. So we have proved $(3.10)$ and (3.11). 
What remains to show is (3.7). We prove that for $\phi(t, \mathbf{x}) \in C_{0}\left(\Omega_{T, R}\right)$,

$$
\lim _{a \rightarrow \infty} \int_{0}^{T} \int_{\mathbb{R}^{d}} y_{a}^{(p)}(t, \mathbf{x}) \phi(t, \mathbf{x}) d t d \mathbf{x}=\int_{0}^{T} \int_{\mathbb{R}^{d}} x_{p} \phi(t, \mathbf{x}) d t d \mathbf{x}
$$

both $\mathbb{P}$-a.s. and in the $L^{1}$-norm. For $\phi(\mathbf{x}) \in C_{0}\left(\mathbb{R}^{d}\right)$ we have

$$
\begin{aligned}
\int_{\mathbb{R}^{d}} y_{a}^{(p)}(t, \mathbf{x}) \phi & (\mathbf{x}) d \mathbf{x} \\
& =\int_{\mathbb{R}^{d}} y_{a}^{(p)}(0, \mathbf{x}) \phi(\mathbf{x}) d \mathbf{x}+\sum_{i, j=1}^{d} \int_{0}^{t} d s \int_{\mathbb{R}^{d}} a_{i j} e_{j}^{(p)} \partial_{x_{j}} \phi d \mathbf{x} .
\end{aligned}
$$

Using the ergodic theorem [3, p. 765], we have

$$
\lim _{a \rightarrow \infty} \int_{\mathbb{R}^{d}} y_{a}^{(p)}(0, \mathbf{x}) \phi(\mathbf{x}) d \mathbf{x}=\int_{\mathbb{R}^{d}} x_{p} \phi(\mathbf{x}) d \mathbf{x},
$$

both $\mathbb{P}$-a.s. and in the $L^{1}$-norm. For fixed $t>0$ the second term on the right hand side of $(3.20)$ tends $\left(\mathbb{P}\right.$-a.s. and in $\left.L^{1}\right)$ to

$$
\sum_{i, j=1}^{d}\left(a_{i j}, e_{j}^{(p)}\right)_{L^{2}} \int_{0}^{t} d s \int_{\mathbb{R}^{d}} \partial_{x_{i}} \phi(\mathbf{x}) d \mathbf{x}
$$

which equals zero, for any $\phi \in C_{0}\left(\mathbb{R}^{d}\right)$. Now choose any compactly supported $\psi \in C_{0}(\mathbb{R}) ;$ then

$$
\begin{aligned}
\lim _{a \rightarrow \infty} \int \psi(t) d t\left[\int y_{a}^{(p)}(t, \mathbf{x}) \phi(\mathbf{x}) d \mathbf{x}\right] & =\lim _{a \rightarrow \infty} \int \psi(t) d t \int y_{a}^{(p)}(0, \mathbf{x}) \phi(\mathbf{x}) d \mathbf{x} \\
& +\lim _{a \rightarrow \infty} \sum_{i, j=1}^{d} \int_{-R}^{R} \psi(t) d t \int a_{i j}\left(a^{2} s+a \mathbf{x}\right) e_{j}^{(p)}\left(a^{2} s+a \mathbf{x}\right) d \mathbf{x}
\end{aligned}
$$

for some $R>0$. As in the previous case, the last limit is zero.

4. Proof of Theorem 2. It has been shown (see [15, Chapter 1]) that for the validity of the central limit theorem one needs to verify

$$
\lim _{\lambda \rightarrow 0+} \lambda\left\|E_{\lambda}^{(p)}\right\|_{L^{2}}^{2}=0
$$

for any $p=1, \ldots, d$. It follows then, according to [15], that

$$
\lim _{\lambda \rightarrow 0+}\left\|D_{i} E_{\lambda}^{(p)}-e_{i}^{(p)}\right\|_{L^{2}}=0, \quad i=1, \ldots, d .
$$

Let us briefly outline why (4.1) implies CLT. We rewrite the process $\mathbf{x}_{\omega}(t)$ in the form (using (2.3))

$$
x_{\omega}^{(p)}(t)-v_{p} t=\lambda \int_{0}^{t} E_{\lambda}^{(p)}\left(\xi_{s}\right) d s-\int_{0}^{t} \mathcal{L} E_{\lambda}^{(p)}\left(\xi_{s}\right) d s+\sum_{q=1}^{d} \int_{0}^{t} \sigma_{p q}\left(\xi_{s}\right) d w_{q}(s) .
$$


Now using the Itô formula we have

$$
x_{\omega}^{(p)}(t)-v_{p} t=\lambda \int_{0}^{t} E_{\lambda}^{(p)}\left(\xi_{s}\right) d s+E_{\lambda}^{(p)}\left(\xi_{0}\right)-E_{\lambda}^{(p)}\left(\xi_{t}\right)+M_{\lambda, t}^{(p)}
$$

where

$$
M_{\lambda, t}^{(p)}=\sum_{q, r=1}^{d} \int_{0}^{t}\left(D_{r} E_{\lambda}^{(p)}\left(\xi_{s}\right)+\delta_{r}^{p}\right) \sigma_{r q}\left(\xi_{s}\right) d w_{q}(s)
$$

and $\delta_{r}^{p}$ denotes the Kronecker symbol. Dividing both sides of (4.3) by $\sqrt{t}$ and taking $\lambda=1 / \sqrt{t}$ we can use (4.1) to argue that the terms corresponding to the first three terms on the right hand side of (4.3) vanish as $t \rightarrow \infty$. The weak convergence of $(1 / \sqrt{t}) M_{1 / \sqrt{t}, t}^{(p)}$ follows from the CLT for martingales. We use the martingale CLT in the version of Helland (see [4, Theorem 5.4]) adapted to our situation. It deals with the martingales admitting jumps so the convergence claimed there is in the sense of the Stone topology on the Skorokhod space $D\left([0, \infty) ; \mathbb{R}^{d}\right)$. The statement of the theorem, modified for the case of martingales with continuous trajectories considered here, can be read as follows.

THEOREM 4. Let $\left(M_{\lambda}^{(1)}(t), \ldots, M_{\lambda}^{(d)}(t)\right), t \geq 0$, be a family of square integrable, continuous trajectory, $\mathbb{R}^{d}$-valued martingales indexed by a parameter $\lambda>0$. Denote by $\left(\mathcal{F}_{\lambda}(t)\right)_{t \geq 0}, \lambda>0$, the filtration that corresponds to a given martingale. Suppose further that the quadratic covariations of martingales satisfy

(i) $\left\langle M_{\lambda}^{(j)}, M_{\lambda}^{(j)}\right\rangle(t) \rightarrow \int_{0}^{t} f_{j}^{2}(s) d s$ as $\lambda \rightarrow 0+$ in $\mathbb{P}$ probability for all $t>0$, where $f_{j}$ is a measurable, non-negative function such that $\int_{0}^{t} f_{j}^{2}(s) d s<\infty$ for all $t>0$.

(ii) $\left\langle M_{\lambda}^{(i)}, M_{\lambda}^{(j)}\right\rangle(t) \rightarrow 0$ for all $t>0$ and $i \neq j$.

Then

$$
\left(M_{\lambda}^{(1)}(t), \ldots, M_{\lambda}^{(d)}(t)\right) \Rightarrow\left(Y^{(1)}(t), \ldots, Y^{(d)}(t)\right)
$$

as $\lambda \rightarrow 0+$, where $Y^{(j)}(t)=\int_{0}^{t} f_{j}(s) d w_{j}(s)$ and $w_{1}, \ldots, w_{d}$ are independent standard Brownian motions. The convergence here is the convergence of stochastic processes with continuous trajectories.

We apply the above theorem in the following way. Let us define $M_{\lambda}^{(p)}(t):=$ $\lambda M_{\lambda, t / \lambda^{2}}^{(p)}, p=1, \ldots, d$, and

$$
N_{\lambda}^{(p)}(t):=\sum_{q=1}^{d} c_{p q} M_{\lambda}^{(q)}(t), \quad p=1, \ldots, d,
$$


where $C:=\left[c_{p q}\right]=\left[D^{*}\right]^{-1 / 2}$ (from Remark 3 it is clear that $C$ exists). Using (4.2) and the mean ergodic theorem we conclude immediately that

$$
\left\langle N_{\lambda}^{(i)}, N_{\lambda}^{(j)}\right\rangle(t) \rightarrow \delta_{i, j} t \quad \text { as } \lambda \rightarrow 0
$$

Applying Theorem 4 we infer that the laws of the martingales $\left(N_{\lambda}^{(1)}(t), \ldots\right.$ $\left.\ldots, N_{\lambda}^{(d)}(t)\right), t \geq 0$, converge weakly, as $\lambda \rightarrow 0+$, to the law of a standard $d$-dimensional Brownian motion. Hence, the laws of $\left(M_{\lambda}^{(1)}(t), \ldots, M_{\lambda}^{(d)}(t)\right)$ converge to the law of a Brownian motion with zero mean and covariance matrix $D^{*}$. In the particular case when $t=1$ and $\lambda=1 / \sqrt{t}$ we obtain the weak convergence of the laws of $(1 / \sqrt{t})\left(M_{1 / \sqrt{t}, t}^{(1)}, \ldots, M_{1 / \sqrt{t}, t}^{(d)}\right)$ as $t \rightarrow \infty$ to the law of a normal vector with mean zero and covariance $D^{*}$.

From (2.5) it follows that (4.1) holds if we show the energy identity

$$
\sum_{i, j=1}^{d}\left(a_{i j} e_{j}^{(p)}, e_{i}^{(p)}\right)_{L^{2}}=-\sum_{i=1}^{d}\left(a_{i p}, e_{i}^{(p)}\right)_{L^{2}}
$$

Now we prove that (4.5) is a consequence of (2.7) and (2.9). Without any loss of generality we may assume that $\mathbf{v}=(1,0, \ldots, 0)$. Our goal is to use an appropriate test function in equation (2.7).

Let $h(\mathbf{x})$ be compactly supported $C^{\infty}$ function such that $\int_{\mathbb{R}^{d}} h(\mathbf{x}) d \mathbf{x}=1$. We also assume that the support of $h$ is contained in $[0,1]^{d}$. In order to use (2.9) we make a suitable scaling. Define

$$
h_{a}(\mathbf{x}):=\frac{1}{a^{d+1}} h\left(\frac{x_{1}}{a^{2}}, \frac{x_{2}}{a}, \ldots, \frac{x_{d}}{a}\right), \quad \mathbf{x} \in \mathbb{R}^{d}
$$

for any $a>0$. Henceforth we will use the vector-like notation $\widetilde{a}:=\left(a^{2}, a, \ldots, a\right)$ where $\widetilde{a}$ has $d$-coordinates, and $\mathbf{x} / \widetilde{a}:=\left(x_{1} / a^{2}, x_{2} / a, \ldots, x_{d} / a\right)$. Now we define the function $\phi_{a}(\mathbf{x} ; \omega):=E^{(p)}(\mathbf{x} ; \omega) h_{a}(\mathbf{x})$ to be used in (2.7). The first term on the left hand side of (2.7) then equals

$$
\frac{1}{2 a^{d+1}} \sum_{i, j=1}^{d} \int_{\mathbb{R}^{d}} \int_{\Omega} a_{i j}\left(T_{\mathbf{x}} \omega\right) \partial_{x_{j}} E^{(p)}(\mathbf{x} ; \omega) \partial_{x_{i}} E^{(p)}(\mathbf{x} ; \omega) h\left(\frac{\mathbf{x}}{\widetilde{a}}\right) d \mathbf{x} \mathbb{P}(d \omega)
$$

$$
+\frac{1}{2 a^{d+1}} \sum_{i, j=1}^{d} \int_{\mathbb{R}^{d}} \int_{\Omega} a_{i j}\left(T_{\mathbf{x}} \omega\right) \partial_{x_{j}} E^{(p)}(\mathbf{x} ; \omega) E^{(p)}(\mathbf{x} ; \omega) \partial_{x_{i}}\left[h\left(\frac{\mathbf{x}}{\widetilde{a}}\right)\right] d \mathbf{x} \mathbb{P}(d \omega)
$$

Taking the limit as $a \rightarrow \infty$ in the first term of (4.6) and using (ii) and the ergodic theorem, we get the term on the left hand side of (4.5). For the second term of (4.6), note that $\partial_{x_{1}}\left[h_{a}(\mathbf{x} / \widetilde{a})\right]=a^{-2}\left(\partial_{x_{1}} h_{a}\right)(\mathbf{x} / \widetilde{a})$ and $\partial_{x_{i}}\left[h_{a}(\mathbf{x} / \widetilde{a})\right]=a^{-1}\left(\partial_{x_{i}} h_{a}\right)(\mathbf{x} / \widetilde{a})$ for $i=2, \ldots, d$. Therefore this term can be 
estimated, with the help of the Cauchy-Schwarz inequality, by

$$
\frac{d}{a^{d+1}} \max _{i, j}\left(\left\|a_{i j}\right\|_{\infty}\left\|e_{j}^{(p)}\right\|_{L^{2}}\right) \sum_{i=1}^{d} \sup _{\mathbf{x} \in C(\mathbf{v}, a)} \frac{\left\|E^{(p)}(\mathbf{x}, \cdot)\right\|_{L^{2}}}{a} \int_{\mathbb{R}^{d}}\left|\left(\partial_{x_{i}} h\right)\left(\frac{\mathbf{x}}{\widetilde{a}}\right)\right| d \mathbf{x},
$$

which vanishes as $a \rightarrow \infty$. The term on the right hand side of (2.7) equals

$$
\begin{aligned}
& -\frac{1}{2 a^{d+1}} \sum_{i=1}^{d} \int_{\mathbb{R}^{d}} \int_{\Omega} a_{i p}\left(T_{\mathbf{x}} \omega\right) \partial_{x_{i}} E^{(p)}(\mathbf{x} ; \omega) h\left(\frac{\mathbf{x}}{\widetilde{a}}\right) d \mathbf{x} \mathbb{P}(d \omega) \\
& -\frac{1}{2 a^{d+1}} \sum_{i=1}^{d} \int_{\mathbb{R}^{d}} \int_{\Omega} a_{i p}\left(T_{\mathbf{x}} \omega\right) E^{(p)}(\mathbf{x} ; \omega) \partial_{x_{i}}\left[h\left(\frac{\mathbf{x}}{\widetilde{a}}\right)\right] d \mathbf{x} \mathbb{P}(d \omega)
\end{aligned}
$$

and by an analogous argument (as in (4.6)) applied to these terms we obtain the expression on the right hand side of (4.5). What remains yet to be proved is the fact that the second term on the left hand side of (2.7) vanishes as $a \rightarrow \infty$. Recall that $\mathbf{v}=(1,0, \ldots, 0)$ so we can rewrite this term in the form

$$
\begin{aligned}
\frac{1}{a^{d+1}} \iint_{\mathbb{R}^{d}} E^{(p)}(\mathbf{x} ; \omega) \partial_{x_{1}} E^{(p)}(\mathbf{x} ; \omega) h\left(\frac{\mathbf{x}}{\widetilde{a}}\right) d \mathbf{x} \mathbb{P}(d \omega) \\
+\frac{1}{a^{d+1}} \int_{\mathbb{R}^{d}} \int_{\Omega} E^{(p)}(\mathbf{x} ; \omega) E^{(p)}(\mathbf{x} ; \omega) \partial_{x_{1}}\left[h\left(\frac{\mathbf{x}}{\widetilde{a}}\right)\right] d \mathbf{x} \mathbb{P}(d \omega) .
\end{aligned}
$$

Integration by parts in the first term of the above expression gives

$$
\begin{aligned}
\frac{1}{a^{d+1}} \int_{\mathbb{R}^{d}} \int_{\Omega} E^{(p)}(\mathbf{x} ; \omega) \partial_{x_{1}} E^{(p)}(\mathbf{x}, \omega) h\left(\frac{\mathbf{x}}{\widetilde{a}}\right) d \mathbf{x} \mathbb{P}(d \omega) \\
=-\frac{1}{a^{d+1}} \int_{\mathbb{R}^{d}} \int_{\Omega} \partial_{x_{1}}\left[E^{(p)}(\mathbf{x} ; \omega) h\left(\frac{\mathbf{x}}{\widetilde{a}}\right)\right] E^{(p)}(\mathbf{x} ; \omega) d \mathbf{x} \mathbb{P}(d \omega) \\
=-\frac{1}{a^{d+1}} \iint_{\mathbb{R}^{d}} \partial_{x_{1}} E^{(p)}(\mathbf{x} ; \omega) h\left(\frac{\mathbf{x}}{\widetilde{a}}\right) E^{(p)}(\mathbf{x} ; \omega) d \mathbf{x} \mathbb{P}(d \omega) \\
\\
-\frac{1}{a^{d+1}} \int_{\mathbb{R}^{d}} \int_{\Omega} E^{(p)}(\mathbf{x} ; \omega) \partial_{x_{1}}\left[h\left(\frac{\mathbf{x}}{\widetilde{a}}\right)\right] E^{(p)}(\mathbf{x} ; \omega) d \mathbf{x} \mathbb{P}(d \omega) .
\end{aligned}
$$

From the above equality we conclude that

$$
\begin{array}{r}
\frac{2}{a^{d+1}} \int_{\mathbb{R}^{d}} \int_{\Omega} \partial_{x_{1}}\left[E^{(p)}(\mathbf{x} ; \omega) h\left(\frac{\mathbf{x}}{\widetilde{a}}\right)\right] E^{(p)}(\mathbf{x} ; \omega) d \mathbf{x} \mathbb{P}(d \omega) \\
=-\frac{1}{a^{d+1}} \int_{\mathbb{R}^{d}} \int_{\Omega}\left[E^{(p)}(\mathbf{x} ; \omega)\right]^{2}\left(\partial_{x_{1}} h\right)\left(\frac{\mathbf{x}}{\widetilde{a}}\right) d \mathbf{x} \mathbb{P}(d \omega) .
\end{array}
$$


In order to establish (4.5) we need to show that

$$
\frac{1}{a^{d+3}} \int_{\mathbb{R}^{d}} \int_{\Omega}\left[E^{(p)}(\mathbf{x} ; \omega)\right]^{2}\left(\partial_{x_{1}} h\right)\left(\frac{\mathbf{x}}{\widetilde{a}}\right) d \mathbf{x} \mathbb{P}(d \omega)
$$

tends to zero as $a \rightarrow \infty$. Indeed, this expression can be estimated by

$$
\frac{1}{a^{d+1}} \sup _{\mathbf{x} \in C(\mathbf{v}, a)}\left(\frac{\left\|E^{(p)}(\mathbf{x}, \cdot)\right\|_{L^{2}}}{a}\right)^{2} \int_{\mathbb{R}^{d}}\left|\left(\nabla_{\mathbf{x}} h\right)\left(\frac{\mathbf{x}}{\widetilde{a}}\right)\right| d \mathbf{x},
$$

which vanishes as $a \rightarrow \infty$ by virtue of (2.9).

REMARK 2. By the definition of $D^{*}=\left[D_{p p^{\prime}}\right]_{p, p^{\prime}=1}^{d}$ we have

$$
D_{p p^{\prime}}=\lim _{t \rightarrow \infty} \frac{\mathbb{E}\left(M_{1 / \sqrt{t}, t}^{(p)}, M_{1 / \sqrt{t}, t}^{\left(p^{\prime}\right)}\right)}{t} .
$$

By (4.4), the fact that $d w_{q}(s) d w_{r}(s)=\delta_{q r} d s$ and the ergodic theorem we have

$$
D_{p p^{\prime}}=\mathbb{E}\left[\sum_{r, r^{\prime}, q=1}^{d}\left(e_{r}^{(p)}+\delta_{r}^{p}\right) \sigma_{r q}\left(e_{r^{\prime}}^{\left(p^{\prime}\right)}+\delta_{r^{\prime}}^{p^{\prime}}\right) \sigma_{r^{\prime} q}\right] .
$$

Hence, since $\sigma^{2}=A$, we have

$$
D^{*}=\mathbb{E}\left[(E+I) A(E+I)^{T}\right]
$$

where $E=\left[e_{j}^{(i)}(\omega)\right], A=\left[a_{i j}(\omega)\right], I=\left[\delta_{i j}\right], i, j=1, \ldots, d$.

REMARK 3. From (4.7) it is clear that $D^{*} \geq \gamma I$.

5. Proof of Theorem 3. (i) Assume that there are two solutions of (2.10); denote them by $e_{i, 1}^{(p)}, e_{i, 2}^{(p)}$. Define $e_{i, 3}^{(p)}=\frac{1}{2}\left(e_{i, 1}^{(p)}+e_{i, 2}^{(p)}\right)$. Then by $(4.5)$,

$$
\begin{aligned}
\frac{1}{2} \sum_{i, j=1}^{d}\left(a_{i j} e_{j, 1}^{(p)}, e_{i, 1}^{(p)}\right)_{L^{2}}+\frac{1}{2} \sum_{i, j=1}^{d}\left(a_{i j} e_{j, 2}^{(p)}, e_{i, 2}^{(p)}\right)_{L^{2}} & =-\sum_{i=1}^{d}\left(a_{i p}, e_{i, 3}^{(p)}\right)_{L^{2}} \\
& =\sum_{i, j=1}^{d}\left(a_{i j} e_{j, 3}^{(p)}, e_{i, 3}^{(p)}\right)_{L^{2}} .
\end{aligned}
$$

Hence $e_{i, 1}^{(p)}=e_{i, 2}^{(p)} \mathbb{P}$-a.s.

(ii) $\mathrm{By}(2.10), e_{i}^{(p)}(\mathbf{v}) \rightarrow e_{i}^{(p)}(\mathbf{0})$ in the weak $L^{2}$ sense, since the left hand side of (2.10) does not depend on $e_{i}^{(p)}$. By the energy identity (4.5) we have

$$
\lim _{\mathbf{v} \rightarrow \mathbf{0}} \sum_{i, j=1}^{d}\left(a_{i j} e_{j}^{(p)}(\mathbf{v}), e_{i}^{(p)}(\mathbf{v})\right)_{L^{2}}=\sum_{i, j=1}^{d}\left(a_{i j} e_{j}^{(p)}(\mathbf{0}), e_{i}^{(p)}(\mathbf{0})\right)_{L^{2}}
$$


Since

$$
\gamma\left\|e_{j}^{(p)}(\mathbf{v})-e_{j}^{(p)}(\mathbf{0})\right\|_{L^{2}}^{2} \leq \sum_{i, j=1}^{d}\left(a_{i j}\left(e_{j}^{(p)}(\mathbf{v})-e_{j}^{(p)}(\mathbf{0})\right), e_{i}^{(p)}(\mathbf{v})-e_{i}^{(p)}(\mathbf{0})\right)_{L^{2}}
$$

by (A2), we conclude that $\lim _{\mathbf{v} \rightarrow \mathbf{0}}\left\|e_{j}^{(p)}(\mathbf{v})-e_{j}^{(p)}(\mathbf{0})\right\|_{L^{2}}^{2}=0, j=1, \ldots, d$.

Acknowledgments. I would like to thank Professor T. Komorowski for inspiration and many discussions on the subject of the article.

\section{References}

[1] P. Billingsley, Convergence of Probability Measures, 2nd ed., Wiley, 1999.

[2] S. Ethier and T. Kurtz, Markov Processes, Wiley, New York, 1986.

[3] A. Fannjiang and T. Komorowski, An invariance principle for diffusion in turbulence, Ann. Probab. 27 (1999), 751-781.

[4] I. S. Helland, Central limit theorems for martingales with discrete or continuous time, Scand. J. Statist. 9 (1982), 79-94.

[5] T. Komorowski and S. Olla, A note on the central limit theorem for two-fold stochastic random walks in a random environment, Bull. Polish Acad. Sci. Math. 51 (2003), $217-232$.

[6] -, -, On homogenization of time-dependent random flows, Probab. Theory Related Fields 121 (2001), 98-116.

[7] S. M. Kozlov, Averaging of random operators, Math. USSR-Sb. 37 (1980), 167-180.

[8] - Conductivity of two-dimensional random media, Russian Math. Surveys 34 (1979), no. 4, 168-169.

[9] —, The method of averaging and walks in inhomogeneous environments, ibid. 40 (1985), no. 2, 73-145.

[10] S. M. Kozlov, S. M. Zhikov and O. A. Oleĭnik, Averaging of parabolic operators, Trudy Moskov. Mat. Obshch. 45 (1982), 182-236 (in Russian).

[11] U. Krengel, Ergodic Theorems, de Gruyter, Berlin, 1985.

[12] C. Landim, S. Olla and H. T. Yau, Convection-diffusion equation with space-time ergodic random flow, Probab. Theory Related Fields 112 (1998), 203-220.

[13] J. Moser, A Harnack inequality for parabolic differential equations, Comm. Pure Appl. Math. 17 (1964), 101-134.

[14] S. Olla, Homogenization of diffusion processes in random fields, École doctorale de École Polytechnique, 1994.

[15] - Central limit theorems for tagged particles and for diffusion in random environment, in: Random Media, Panorama et Synthèses 12, Soc. Math. France, 2001, $75-100$.

[16] K. Oelschläger, Homogenization of a diffusion process in a divergence-free random field, Ann. Probab. 16 (1988), 1084-1126.

[17] H. Osada, Homogenization of diffusion processes with random stationary coeffcients, in: Probability Theory and Mathematical Statistics, Lecture Notes in Math. 1021, Springer, Berlin, 1983, 507-517.

[18] G. Papanicolaou and S. R. S. Varadhan, Boundary value problems with rapidly oscillating random coefficients, in: Random Fields (Esztergom, 1979), Colloq. Math. Soc. János Bolyai 27, North-Holland, Amsterdam, 1982, 835-873. 
[19] L. C. G. Rogers and D. Williams, Diffusions, Markov Processes and Martingales, Vol. 1, Wiley, 1994.

Ernest Nieznaj

Department of Mathematics

Faculty of Electrical Engineering and Computer Science

Lublin University of Technology

Nadbystrzycka 38A

20-618 Lublin, Poland

E-mail: e.nieznaj@pollub.pl

Received February 11, 2005 